\title{
ON THE THEOREM OF WÓJCIK
}

\author{
by A. ROTKIEWICZ \\ Dedicated to the memory of my friend Jan Wójcik (1936-1994)
}

(Received 13 October, 1994)

In the paper [3] the following lemma was proved.

LemMA. Let $a, b$ and $c$ be positive integers such that $a$ and $b c$ are relatively prime. Then there are infinitely many primes $p$ in the arithmetic progression $a x+b(x=$ $0,1,2, \ldots)$ such that

$$
p \mid\left(2^{(p-1) / c}-1\right)
$$

In 1982 Jan Wójcik proved [10] a similar result about the so called Lehmer numbers. Lehmer numbers can be defined as follows:

$$
P_{n}(\alpha, \beta)= \begin{cases}\left(\alpha^{n}-\beta^{n}\right) /(\alpha-\beta) & \text { if } n \text { is odd, } \\ \left(\alpha^{n}-\beta^{n}\right) /\left(\alpha^{2}-\beta^{2}\right) & \text { if } n \text { is even, }\end{cases}
$$

where $\alpha, \beta$ roots of the trinomial $z^{2}-\sqrt{L} z+M$, its discriminant is $D=L-4 M$ and $L>0$ and $M$ are rational integers. We can assume without any essential loss of generality that $(L, M)=1$.

Put for the moment $P_{n}^{\prime}=P_{n}(\alpha, \beta)$. Lehmer numbers can be also defined as follows

$$
\begin{gathered}
P_{1}^{\prime}=P_{2}^{\prime}=1, \\
P_{n}^{\prime}= \begin{cases}L P_{n-1}^{\prime}-M P_{n-2}^{\prime} & \text { if } n \text { is odd, } \\
P_{n-1}^{\prime}-M P_{n-2}^{\prime} & \text { if } n \text { is even. }\end{cases}
\end{gathered}
$$

In 1982 Jan Wójcik [10] proved the following

THEOREM W. If $\alpha, \beta$ defined above are different from zero and $\alpha / \beta$ is not a root of unity then there exists a positive integer $k_{0}$ such that for every positive integer $k$ divisible by $k_{0}$ and for all positive integers $a$ and $b$, where $(a, b)=1$ and $b \equiv 1(\bmod (a, k))$, there exist infinitely many primes satisfying the conditions

$$
p \equiv b(\bmod a), \quad p \equiv 1(\bmod k), \quad p \mid P_{(p-1) k k}(\alpha, \beta) .
$$

Remark. For any $\alpha, \beta$ in Theorem $\mathrm{W}$, the constant $k_{0}=k_{0}(\alpha, \beta)$ may be given explicitly [11]. For example, for the Fibonacci sequence, $k_{0}=20$.

Here we shall prove a similar result for composite numbers. Let

$$
V_{n}= \begin{cases}\left(\alpha^{n}+\beta^{n}\right) /(\alpha+\beta) & \text { if } n \text { is odd, } \\ \left(\alpha^{n}+\beta^{n}\right) & \text { if } n \text { is even, }\end{cases}
$$

denote the $n$th term of the associated Lehmer recurring sequence. The associated Lehmer sequence $V_{k}$ can be defined as follows: $V_{0}=2, V_{1}=1$, and for $n \geq 2$

$$
V_{n}= \begin{cases}L V_{n-1}-M V_{n-2} & \text { for } n \text { even, } \\ V_{n-1}-M V_{n-2} & \text { for } n \text { odd. }\end{cases}
$$

Glasgow Math. J. 38 (1996) 157-162. 
An odd composite number $n$ is a strong Lehmer pseudoprime with parameters $L, M$ (or for the bases $\alpha$ and $\beta)$ if $(n, D L)=1$ and with $n-(D L / n)=d .2^{s}, d$ odd, where $(D L / n)$ is the Jacobi symbol, we have either

$$
\begin{aligned}
& \qquad P_{d} \equiv 0(\bmod n), \text { or } \\
& V_{d .2^{\prime}} \equiv 0(\bmod n), \text { for some } r \text { with } 0 \leq r<s .
\end{aligned}
$$

Every odd prime $n$ satisfies (i) or (ii) provided $(n, D L)=1$ (cf. [6]).

In 1994 I proved [6] the following

THEOREM T. If $\alpha, \beta$ defined above are different from zero and $\alpha / \beta$ is not a root of unity (that is $\langle L, M\rangle \neq\langle 1,1\rangle,\langle 2,1\rangle,\langle 3,1\rangle)$ then every arithmetical progression $a x+b(x=$ $0,1,2, \ldots)$, where $a, b$ are relatively prime positive integers, contains an infinite number of odd strong Lehmer pseudoprimes for the bases $\alpha$ and $\beta$.

In $1982 \mathrm{I}$ proved [5] this theorem only in the case $D=(\alpha-\beta)^{2}>0$. We shall introduce the following

Definition. Let $P_{n}(\alpha, \beta)$ denote the $n$th Lehmer number. An odd composite $n$ is a $k$ th order strong Lehmer pseudoprime for the bases $\alpha$ and $\beta$ if $(n, D L)=1$ and, with $n-(D L / n) \equiv 0(\bmod k), d=\frac{1}{k}(n-(D L / n)),(d, k)=1$, we have

$$
P_{d}(\alpha, \beta) \equiv 0(\bmod n) .
$$

For $k=2^{s}$ we get a strong Lehmer pseudoprime satisfying (i), for the bases $\alpha$ and $\beta$.

Now we shall prove the following

THEOREM $\mathrm{W}_{1}$. Let $P_{n}(\alpha, \beta)$ denote the nth Lehmer number. If $\alpha / \beta$ is not a root of unity then there exists a positive integer $k_{0}$ such that for every positive integer $k$ divisible by $k_{0}$ and for all positive integers $a$ and $b$, where $(a, b)=1$ and $b \equiv 1+k\left(\bmod k^{2}\right)$, in every arithmetical progression $a x+b(x=0,1,2, \ldots)$ there exist infinitely many $k$ th order strong Lehmer pseudoprimes for the bases $\alpha$ and $\beta$.

For each positive integer $n$ we denote by $\phi_{n}(\alpha, \beta)=\bar{\phi}_{n}(L, M)$ the $n$th cyclotomic polynomial

$$
\bar{\phi}_{n}(L, M)=\phi_{n}(\alpha, \beta)=\prod_{(m, n)=1}\left(\alpha-\zeta_{n}^{m} \beta\right),
$$

where $\zeta_{n}$ is a primitive $n$th root of unity and the product is over the $\varphi(n)$ integers $m$ with $1 \leq m \leq n$ and $(m, n)=1$.

It will be convenient to write

$$
\phi(\alpha, \beta ; n)=\phi_{n}(\alpha, \beta) .
$$

It is easy to see that $\phi(\alpha, \beta ; n)>1$ for $D=L-4 M>0, n>2$. A prime factor $p$ of $P_{n}=P_{n}(\alpha, \beta)$ is called a primitive factor of $P_{n}$ if $p \mid P_{n}$ but $p \nmid D L P_{3} \ldots P_{n-1}$.

Assume that $M \neq 0, D=L-4 M \neq 0,\langle L, M\rangle \neq\langle 1,1\rangle,\langle 2,1\rangle$, $\langle 3,1\rangle$; (i.e. $\beta / \alpha$ is not a root of unity).

The following results are well known. 
Lemma 1. (Lehmer [2]). Let $n \neq 2^{\gamma}, 3 \cdot 2^{\gamma}$. Denote by $r=r(n)$ the largest prime factor of $n$. If $r \nmid \phi(\alpha, \beta ; n)$, then every prime $p$ dividing $\phi(\alpha, \beta ; n)$ is a primitive prime divisor of $P_{n}$.

Every primitive prime divisor $p$ of $P_{n}$ is $\equiv(D L / p)(\bmod p)$. If $r \mid \phi(\alpha, \beta ; n), r^{l} \| n$ (which is to say $r^{l} \mid n$ but $\left.r^{l+1} \nmid n\right)$ then $r \| \phi(\alpha, \beta ; n)$ and $r$ is a primitive prime divisor of $P_{n / r^{\prime}}$

Lemma 2. The number $P_{n}$ for $n>12, D>0$ has a primitive prime divisor (see Durst [1], Ward [9]). If $D<0$ and $\beta / \alpha$ is not a root of unity, then, for $n>n_{0}(\alpha, \beta), P_{n}$ has a primitive prime divisor. The number $n_{0}(\alpha, \beta)$ can be effectively computed (Schinzel [7]); $n_{0}=n_{0}(\alpha, \beta)=e^{452} .4^{67}$ (Stewart [8]). We have $|\phi(\alpha, \beta ; n)|>1$ for $n>n_{0}([7],[8])$.

Lemma 3. (Rotkiewicz [4, Lemma 5]). Let

$$
\psi\left(p_{1}^{\alpha_{1}} p_{2}^{\alpha_{2}} \ldots p_{k}^{\alpha_{k}}\right)=2 p_{1}^{\alpha_{1}} p_{2}^{\alpha_{2}} \ldots p_{k}^{\alpha_{k}}\left(p_{1}^{2}-1\right)\left(p_{2}^{2}-1\right) \ldots\left(p_{k}^{2}-1\right) .
$$

If $q$ is a prime such that $q^{2} \| n$ and $a$ is a natural number such that $a \psi(a) \mid(q-1)$, then $\phi(\alpha, \beta ; n) \equiv 1(\bmod a)$.

Proof of Theorem $W_{1}$. It is sufficient to show that there exists one $k$ th order strong Lehmer pseudoprime for the bases $\alpha$ and $\beta$ of the form $a x+b$. To see this just notice that we then have such pseudoprimes of the shape $a d x+b$ for every natural $d$ with $(d, b)=1$ and we may choose $d$ as large as we wish. We may also suppose without loss of generality that $b$ is odd and that $4 D L \mid a$, since if $b_{1}$ is prime of the form $k^{2} a t+b$, then every term of the progression $k^{2} a t+b_{1}(t=1,2, \ldots)$ is $\equiv b(\bmod a)$, its difference is $k^{2} a$ and $\left(k^{2} a, b_{1}\right)=1$.

Let $D L k_{0} \mid k$ where $k_{0}$ is an integer from the theorem of Wójcik. We have $b_{1}=k^{2} a t+b \equiv 1+k\left(\bmod k^{2}\right)$. Now let $p_{1}, p_{2}, p_{3}, p_{4}$ be different primes such that $\left(p_{1} p_{2} p_{3} p_{4}, a k\right)=1$ and let $q$ be a prime number such that

$$
c \psi(c) \mid q-1, \quad c=k^{2} a p_{1} p_{2} p_{3} p_{4} .
$$

Let $m$ be a positive integer such that

$$
\begin{aligned}
& m \equiv b\left(\bmod a k^{2}\right), \\
& m \equiv 1+p_{1} p_{2} p_{3} p_{4} q^{2}\left(\bmod p_{1}^{2} p_{2}^{2} p_{3}^{2} p_{4}^{2} q^{3}\right) .
\end{aligned}
$$

Such positive $m$ exists by the Chinese Remainder Theorem. From (4) and $b \equiv 1+$ $k\left(\bmod k^{2}\right)$, it follows that

$$
\left(m, a k^{2} p_{1}^{2} p_{2}^{2} p_{3}^{2} p_{4}^{2} q^{3}\right)=1 .
$$

Since $m \equiv b \equiv 1+k\left(\bmod k^{2}\right)$ we have $m \equiv 1(\bmod k)$. Thus also $m \equiv$ $1\left(\bmod \left(k^{2} a p_{1}^{2} p_{2}^{2} p_{3}^{2} p_{4}^{2} q^{2}, k\right)\right)$ and by, Theorem $\mathrm{W}$, there exist infinitely many primes $p$ in the arithmetical progression $k^{2} a p_{1}^{2} p_{2}^{2} p_{3}^{2} p_{4}^{2} q^{3} x+m(x=1,2, \ldots)$ for which

$$
P_{(p-1) / k}(\alpha, \beta) \equiv 0(\bmod p) \text {. }
$$

Let $p$ be one of them. From $4 D L k_{0} \mid k, m \equiv 1(\bmod k)$ it follows that $m \equiv 1(\bmod 4 D L)$, hence $(D L / m)=1$ and also $(D L / p)=1$. We have that $(p-1) / k \equiv(m-1) / k \equiv 1(\bmod k)$, hence $((p-1) / k, k)=1$.

Let $\bar{r}$ denote the greatest prime factor of $p-1$. It is easy to see that one of numbers 
$\phi\left(\alpha, \beta ;(p-1) / k p_{i}\right)$ for $i=1,2,3,4$ can be divisible by $\bar{r}$ and only one can be divisible by $p$. Without loss of generality we can assume that $p \nmid \phi\left(\alpha, \beta ;(p-1) / k p_{i}\right)$ and $\bar{r} \nmid \phi\left(\alpha, \beta ;(p-1) / k p_{i}\right)$ for $i=1,2$.

Let $m_{i}=\phi\left(\alpha, \beta ; \frac{p-1}{k p_{i}}\right)$ for $i=1,2$. Now we shall prove that if $m_{1}>0$ or $m_{2}>0$ then $p m_{1}$ or $p m_{2}$ is our required pseudoprime and if $m_{1}<0$ and $m_{2}<0$ then $p m_{1} m_{2}$ is our required pseudoprime. First we shall consider the case when $m_{1}>0$ or $m_{2}>0$.

Suppose for example that $m_{1}>0$. Let $s_{1}=\frac{p-1}{k p_{1}}$. By Lemma 1 every prime factor $t$ of $m_{1}$ is congruent to $(D L / t)\left(\bmod s_{1}\right)$. Since $m_{1}>0$, by Lemma $2, m_{1}$ is a positive integer greater than 1 . So

$$
m_{1} \equiv\left(D L / m_{1}\right)\left(\bmod s_{1}\right) \text {. }
$$

Certainly $q^{2} \| s_{1}=(p-1) / k p_{1}$. So from $a \psi(a)|(q-1), 4 D L| a$, by Lemma 3 we have $m_{1} \equiv 1(\bmod 4 D L)$. So $\left(D L / m_{1}\right)=1$ and from $(5)$ it follows that

$$
m_{1} \equiv 1\left(\bmod s_{1}\right), \quad s_{1}=\frac{p-1}{k p_{1}} .
$$

Further, from $q^{2} \| s_{1}, k p_{1} \psi\left(k p_{1}\right) \mid(p-1)$, by Lemma 3 we have

$$
m_{1} \equiv 1\left(\bmod k p_{1}\right) \text {. }
$$

Since $p \equiv 1+k\left(\bmod k^{2}\right)$ and $p \equiv 1+p_{1} p_{2} p_{3} p_{4} q^{2}\left(\bmod p_{1}^{2}\right)$, we have $\left(s_{1}, k p_{1}\right)=1$. Thus from (6) and (7) we get

$$
m_{1} \equiv 1(\bmod (p-1))
$$

and $n_{1}=p m_{1} \equiv 1(\bmod (p-1)) ;$ hence

$$
\left(n_{1}-1\right) / k \equiv 0(\bmod (p-1) / k) .
$$

From $k^{2} \psi\left(k^{2}\right)\left|(q-1), q^{2}\right|(p-1) / k$, by Lemma 3 we get

$$
m_{1} \equiv 1\left(\bmod k^{2}\right)
$$

hence $n_{1}=p m_{1} \equiv(1+k) 1 \equiv 1+k\left(\bmod k^{2}\right)$ and

$$
\left(\left(n_{1}-1\right) / k, k\right)=1 .
$$

Further, $\left(D L / n_{1}\right)=\left(D L / p m_{1}\right)=(D L / p)\left(D L / m_{1}\right)=1.1=1$. Thus from (9) and (11) we get

$$
m_{1}=\phi\left(\alpha, \beta ;(p-1) / k p_{1}\right)\left|P_{(p-1) / k}\right| P_{\left(n_{1}-1\right) / k}=P_{\left(n_{1}-\left(D L / n_{1}\right)\right) / k},
$$

where $\left(\left(n_{1}-\left(D L / n_{1}\right)\right) / k, k\right)=1, P_{i}=P_{i}(\alpha, \beta)$.

Also

$$
p\left|P_{(p-1) / k}\right| P_{\left(n_{1}-\left(D L / n_{1}\right)\right) / k}
$$

Since $\left(p_{1}, m_{1}\right)=1$, by (12) and (13) we have

$$
n_{1}=p m_{1} \mid P_{\left(n_{1}-\left(D L / n_{1}\right)\right) / k}
$$




\section{ON THE THEOREM OF WÓJCIK}

Since $m_{1} \equiv 1(\bmod a)$ we have

as required.

$$
n_{1}=p m_{1} \equiv b .1 \equiv b(\bmod a)
$$

If the both numbers $m_{1}$ and $m_{2}$ are negative their product $m_{12}$ is positive and

$$
m_{12} \equiv\left(D L / m_{12}\right)\left(\bmod (p-1) / k p_{1} p_{2}\right),
$$

where $m_{12}=m_{1} m_{2}, m_{i}=\phi\left(\alpha, \beta ;(p-1) / k p_{i}\right.$ for $i=1,2$.

Indeed, let $m_{12}=q_{1}^{\alpha_{1}} q_{2}^{\alpha_{2}} \ldots q_{l}^{\alpha_{t}}$. By Lemma 1 we have

Thus

$$
q_{i}^{\alpha_{i}} \equiv\left(D L / q_{i}\right)^{\alpha_{i}}\left(\bmod (p-1) / k p_{1} p_{2}\right) .
$$

$$
\begin{aligned}
m_{12} & \equiv\left(D L / q_{1}\right)^{\alpha_{1}}\left(D L / q_{2}\right)^{\alpha_{2}} \ldots\left(D L / q_{l}\right)^{\alpha_{1}} \equiv\left(D L / q_{1}^{\alpha_{1}}\right)\left(D L / q_{2}^{\alpha_{2}}\right) \ldots\left(D L / q_{l}^{\alpha_{1}}\right) \\
& \equiv\left(D L / m_{12}\right)\left(\bmod (p-1) / k p_{1} p_{2}\right) .
\end{aligned}
$$

Certainly $q^{2} \|(p-1) / k p_{1} p_{2}$ and $a \psi(a) \mid q-1$ and by Lemma $3, m_{1} \equiv 1(\bmod a)$ for $i=1,2$; hence we have $m_{12} \equiv 1(\bmod a)$.

Since $4 D L \mid a$, we have $m_{12} \equiv 1(\bmod 4 D L)$. So $\left(D L / m_{12}\right)=1$ and from $(15)$ we get

$$
m_{12} \equiv 1\left(\bmod (p-1) / k p_{1} p_{2}\right) \text {. }
$$

From $p_{1} p_{2} \psi\left(p_{1} p_{2}\right)\left\|(q-1), q^{2}\right\|(p-1) / k p_{1} p_{2}$, by Lemma 3 we have $m_{i} \equiv 1\left(\bmod p_{1} p_{2}\right)$ for $i=1,2$; hence

$$
m_{12} \equiv 1\left(\bmod p_{1} p_{2}\right)
$$

Since $p_{1}\left\|(p-1), p_{2}\right\|(p-1)$, from (16) and (17) we get

$$
m_{12} \equiv 1(\bmod (p-1) / k) \text {. }
$$

From $k^{2} \psi\left(k^{2}\right) \mid(q-1), q^{2} \|(p-1) / k p_{i}$, by Lemma 3 we get $m_{i} \equiv 1\left(\bmod k^{2}\right)$; hence $m_{12}=m_{1} \cdot m_{2} \equiv 1\left(\bmod k^{2}\right), n_{12}=p m_{12} \equiv 1+k\left(\bmod k^{2}\right)$. Hence $\left(\left(n_{12}-1\right) / k, k\right)=1$. Also $\left(D L / n_{12}\right)=1\left(\right.$ recall that $\left.\left(D L / m_{1}\right)=1, p \equiv 1(\bmod 4 D L)\right)$. By Lemma $2, m_{12}>1$ and

$$
m_{12}=\phi\left(\alpha, \beta ;(p-1) / k p_{1}\right) \cdot \phi\left(\alpha, \beta ;(p-1) / k p_{2}\right)\left|P_{(p-1) / k}\right| P_{\left(n_{12}-1\right) / k}=P_{\left(n_{12}-\left(D L / n_{12}\right)\right) / k} \text {. }
$$

Also

and since $\left(p, m_{12}\right)=1$ we have

$$
p\left|P_{(p-1) / k}\right| P_{\left(n_{12}-\left(D L / n_{12}\right)\right) / k}
$$

where

$$
n_{12}=m_{12} p \mid P_{\left(n_{12}-\left(D L / n_{12}\right)\right) / k},
$$

$$
\left.\left(n_{12}-\left(D L / n_{12}\right)\right) / k, k\right)=1 \text { and } n_{12}=p m_{12} \equiv a \cdot 1 \equiv b(\bmod a)
$$

as required.

\section{REFERENCES}

1. L. K. Durst, Exceptional real Lehmer sequences, Pacific J. Math. 9 (1959), 437-441.

2. D. H. Lehmer, An extended theory of Lucas functions, Ann. Math. (2) 31 (1930), 419-448. 83-86.

3. A. Rotkiewicz, On the prime factors of the number $2^{p-1}-1$, Glasgow Math. J. 9 (1968),

4. A. Rotkiewicz, On the pseudoprimes of the form $a x+b$ with respect to the sequence of Lehmer, Bull. Acad. Polon. Sci. Ser. Sci. Math. Astronom. Phys. 20 (1972), 349-354. 
5. A. Rotkiewicz, On Euler Lehmer pseudoprimes and strong Lehmer pseudoprimes with parameters $L, Q$ in arithmetic progressions, Math. Comp. 39 (1982), 239-247.

6. A. Rotkiewicz, On strong Lehmer pseudoprimes in the case of negative discriminant in arithmetic progression, Acta Arith. 68 (1994), 145-151.

7. A. Schinzel, The intrinsic divisors of Lehmer numbers in the case of negative discriminant, Ark. Math. 4 (1962), 413-416.

8. C. L. Stewart, Primitive divisors of Lucas and Lehmer numbers, Transcendence Theory: Advances and Application (Academic Press, 1977), 79-92.

9. M. Ward, The intrinsic divisors of Lehmer numbers, Ann. Math. (2) 62 (1955), 230-236. $155-174$.

10. J. Wójcik, Contribution to the theory of Kummer extension, Acta Arith. 40 (1982),

11. J. W6jcik, On the density of some sets of primes connected with cyclotomic polynomials, Acta Arith. 41 (1982), 117-131.

INSTITUTE OF MATHEMATICS

Polish ACAdemy of SCIEnces

UL. ŚNIADECKICH 8

00-950 Warszawa, Poland

AND

TeChNical University in Biatystok

ul. Wiejska 45, 15-351 Biatystok, Poland 